\title{
A lightweight magnetic gripper for an aerial delivery vehicle: design and applications
}

\author{
Giuseppe Sutera ${ }^{1}$, Dario Calogero Guastella ${ }^{1}$, Giovanni Muscato ${ }^{1,2}$ \\ ${ }^{1}$ Dipartimento di Ingegneria Elettrica Elettronica e Informatica, University of Catania, Catania, Italy \\ ${ }^{2}$ Istituto di Calcolo e Reti ad Alte Prestazione, Consiglio Nazionale delle Ricerche, ICAR-CNR, Palermo, Italy
}

\begin{abstract}
In recent years, drones have become widely used in many fields. Their vertical flight capability makes these systems suitable for carrying out a variety of tasks. In this paper, the delivery service they provide is analysed. The delivery of goods quickly and to remote areas is a relevant application scenario; however, the systems proposed in the literature use electromagnets, which affect the duration of the flight. In addition, these devices are heavy and suffer from high energy consumption, which reduces the maximum transportable payload. This study proposes a new lightweight magnetic plate composed of permanent magnets, capable of collecting and positioning any object as long as it has a ferromagnetic surface on the top. This plate was developed for the Mohamed Bin Zayed International Robotics Challenge 2020, an international robotics competition for multi-robot systems. Challenge two of this competition required a drone capable of picking up different types of bricks and assembling them to build a wall according to an assigned pattern. The bricks were of different colours and sizes, with weights ranging from 1 to $2 \mathrm{~kg}$. In light of this, it was concluded that weight was the most relevant specification to consider in drone design.
\end{abstract}

\section{Section: RESEARCH PAPER}

Keywords: Low-power gripper; pick and place; rapid prototyping; permanent magnets; mobile robot application

Citation: Giuseppe Sutera, Dario Calogero Guastella, Giovanni Muscato, A lightweight magnetic gripper for a delivery aerial vehicle: design and applications, Acta IMEKO, vol. 10, no. 3, article 10, September 2021, identifier: IMEKO-ACTA-10 (2021)-03-10

Section Editor: Bálint Kiss, Budapest University of Technology and Economics, Hungary

Received January 15, 2021; In final form September 6, 2021; Published September 2021

Copyright: This is an open-access article distributed under the terms of the Creative Commons Attribution 3.0 License, which permits unrestricted use, distribution, and reproduction in any medium, provided the original author and source are credited.

Funding: This research was partially funded by the 'Safe and Smart Farming with Artificial Intelligence and Robotics - programma ricerca di ateneo UNICT 2020 -- 22 linea 2' project.

Corresponding author: Giuseppe Sutera, e-mail: giuseppe.sutera@unict.it

\section{INTRODUCTION}

Unmanned aerial vehicles or drones represent the future of many evolving sectors. Autonomous delivery is one of these, and this sector is developing rapidly thanks to new platforms that allow the transportation of weights heavier than that of the platforms themselves. These vehicles are often used to transport wares to areas that are difficult to reach quickly using standard means of transport. In order to make the entire delivery process autonomous, it is necessary to use an easily coupling system to attach the package to the drone. In the literature, different techniques have been described, which will be analysed in detail in this paper.

The snap-fit method requires a high level of accuracy in positioning, as there are a number of fixing pins that must be inserted perfectly into the relevant holes. Adhesion is an excellent solution for picking up and placing objects with metal parts. For this reason, electro-adhesion [1] and electromagnets [2]-[4] have been analysed, which represent a valid choice in terms of ease of coupling, but they require a high operating current to create a magnetic field. In terms of energy consumption, this can cause a reduction in flight time. In this approach [3], the use of an electro-permanent magnet reduces energy absorption since it requires a current only in the release phase. However, the typical shape and weight of these devices require the design of bulky housings that do not allow a plate to be used that is suitable for the intended purpose. For the present study, a plate was developed in accordance with the specifications of the 'Mohamed Bin Zayed International Robotics Challenge 2020' (MBZIRC $^{1}$ ). One of the challenges in this competition was to create a drone capable of lifting different types of bricks off the

\footnotetext{
1 Website: https://www.mbzirc.com/.
} 
ground and arranging them to build a wall according to an assigned pattern. For this purpose, a magnetic plate was created using passive magnets inserted inside a 3D-printed structure. The release system was incorporated into the plate and consisted of several levers operated by a single servo control. This setup allows the detachment of ferromagnetic objects without high energy consumption and requires a power supply only in the release phase in order to activate the servomotor. Furthermore, thanks to its design, the developed gripper allows the operators to optimise the weight and energy consumption while still guaranteeing a lifting capacity exceeding $2 \mathrm{~kg}$.

\section{MECHANICAL DESIGN}

The prototype in this study had a flat profile and an attractive force comparable to a commercial device, despite its lightness. The supporting structure was built with rapid prototyping techniques using the Zortrax M200 printer, which is equipped with an extruder with a $0.4 \mathrm{~mm}$ nozzle and a layer resolution of 90-390 microns. Of the materials available, Z-ULTRAT was selected for this project, a blend of Zortrax filaments created to enhance the properties of acrylonitrile butadiene styrene (ABS) in terms of durability.

The entire printing process took about 20 hours, to which 30 minutes were added to integrate the servomotor and the other mechanical parts (such as pins, ball bearings and screws). The considerable difference between printing and assembly times prompted a search for a solution to speed up the printing process. It was therefore decided to divide the prototype into several smaller components. Although this solution increased the overall printing time by about $25 \%$ (due to the increased number of prints and, hence, of printer initialisations), each component part had a printing time ranging from 30 to 120 minutes.

The resulting modular structure of the prototype made it quick to repair. As will be explained later, the bottom part of the plate was the area that was exposed the most to wear, as it was in contact with the ground and the ferrous coupling surfaces.

The dimensions of the realised prototype fulfilled the specific requirements of the MBZIRC 2020 competition, where the magnetic plate was tested. The setup used for the competition had a length of $15 \mathrm{~cm}$, a width of $10 \mathrm{~cm}$, a height of $5 \mathrm{~cm}$ and weighed $195 \mathrm{~g}$. However, the modularity of the prototype made it possible to reduce the contact surface to $9 \times 9 \mathrm{~cm}$ so that it was suitable for smaller drones while still maintaining the same supporting structure.

The plate consisted of four pieces, two for each of the two layers that composed it. The first layer was $0.6 \mathrm{~cm}$ high and consisted of two smaller pieces that were connected with a dovetail joint (Figure 1). During the design phase, a set of beams was designed with a two-fold purpose: 1 ) increasing the rigidity to compensate for the thinness and 2) creating the slots in which to insert the magnets and the supports for the release levers (Figure 2). Two commercial magnets were tested with the same width $(10 \mathrm{~mm})$ and length $(20 \mathrm{~mm})$ but different heights $(2$ and $5 \mathrm{~mm}$ ) and, hence, different attraction forces (Table 1).

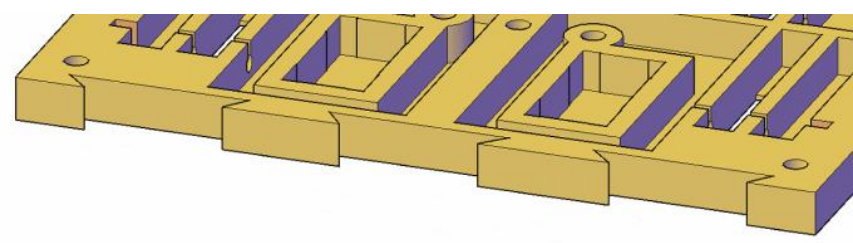

Figure 1. Mechanical dovetail interlocking.

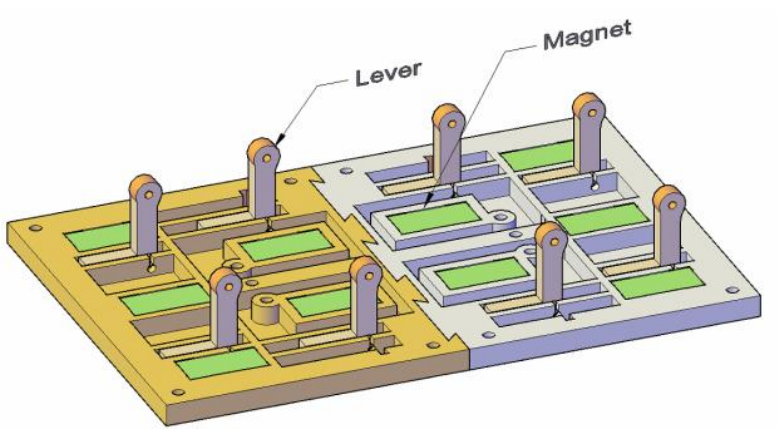

Figure 2. Structure with reinforcement beams, release levers and magnets (in green).

The design choice of creating a covering layer in the lower part made the external surface smooth and free of friction. The magnets were securely fixed in custom housings that prevented them from coming out once the object to be lifted had been hooked (Figure 3).

The release system was operated by a servomotor located in a central position in order to guarantee the centring of the weight. Once activated by a digital pin from flight control unit (FCU) the rotation starts by sending a PWM signal, from rest position up to release position and back. An MG995 commercial servomotor was used, capable of developing a torque of $10 \mathrm{~kg} / \mathrm{cm}(6 \mathrm{~V})$ on the shaft required to operate the cascade of L-shaped levers, located along the length of the plate, to move and push the object away from the magnets. The increase in distance produced a decrease in the force of attraction, and the object is released by gravity. The release phase lasts 1 second. During this period the servomotor draws $600 \mathrm{~mA}$, after which the motor returns to the rest position where the consumption is reduced to $10 \mathrm{~mA}$.

In the proposed solution, neodymium magnets are used for their capability to attract ferrous surfaces. The operating principle was based on the force of attraction, which follows the equation below:

$$
F=\frac{B^{2} A}{2 \mu_{0}},
$$

where $F$ is the force in $\mathrm{N}, A$ is the surface area of the magnetic pole in $\mathrm{m}^{2}, B$ is the magnetic flux density in Tesla and $\mu_{0}$ is the permeability of air.

The second law of dynamics defines the force of gravity proportional to the mass $\mathrm{m}$, therefore $F=m \cdot g$. From this it follows that the lifting mass $m$ is given by

$$
m=\frac{B^{2} A}{2 \mu_{0} g} \text {. }
$$



Figure 3. Cross-section view. 
Table 1. Overview of the properties of the magnets used.

\begin{tabular}{lcc}
\hline Property & Magnet 1 & Magnet 2 \\
\hline Material & NdFeB & NdFeB \\
Weight & $3.04 \mathrm{~g}$ & $7.6 \mathrm{~g}$ \\
Shape & Parallelepiped & Parallelepiped \\
Dimensions & $20 \times 10 \times 2 \mathrm{~mm}$ & $20 \times 10 \times 5 \mathrm{~mm}$ \\
Surface of the poles & $20 \times 10 \mathrm{~mm}$ & $20 \times 10 \mathrm{~mm}$ \\
Coating & Nickel plated & Nickel plated \\
Magnetisation & N45 & N45 \\
Force of attraction & about 2.1 kg & about 3.8 kg \\
\hline
\end{tabular}

Another relevant factor for this approach is the speed of grip and release. During the development of the drone, there was a focus on the coupling speed because, during the approach phase, the drone had to fly over an object placed on the ground and lift it up. During the preliminary tests, two issues were observed when flying close to the ground: 1) the displacement of the object and 2) the instability of the drone. Both issues were ascribed to the rotors' downwash [5], which is the air flow generated by the propulsion system. In the first case, the object to be grabbed moved when the drone flew a few centimetres above it, so it was necessary for the drone to ascend and repeat the grabbing procedure. This repositioning, in view of the challenge, increased the time required to complete the task. Furthermore, the instability caused by the turbulence of the propulsion system interfered with the final manoeuvres needed for the proper positioning of the object. The use of an electromagnet would have introduced a further delay due to the need to maintain the position of the object near the ferrous surface during activation. Driven by the need to reduce operating times, the choice of using permanent magnets, which are constantly 'active', also allowed for a reduction in this additional latency. During the release phase, this problem did not arise, and it was possible to drop the object even at a short distance from the ground.

Another relevant aspect that required specific investigation was the coupling system between the drone and the magnetic plate. Two approaches were tested, one consisting of a sliding prismatic-like joint with a cardan system near the plate and a damped system, which was the final solution adopted. In the first approach, shown in Figure 4, the variable length of the sliding joint allowed the drone to pick up objects up to $50 \mathrm{~cm}$ below its flight altitude. However, the considerable variation in the centre of mass once the object was gripped caused oscillations that increased energy consumption, as the autopilot had to continuously correct the vehicle's position. The final proposed solution consisted of four cables tensioned by as many springs,

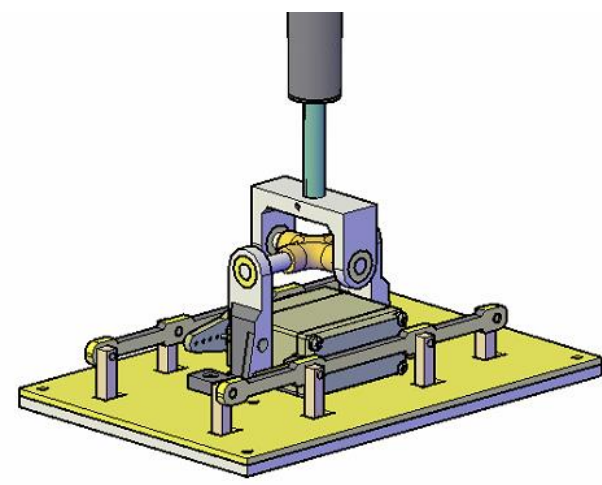

Figure 4. Approach with sliding prismatic-like joint with a cardan system near the plate. thus dampening the mechanical shocks and vibrations on the object due to oscillations during transportation. Furthermore, the cables allowed the plate to be attracted to the metal sheet on the object, as the plate could move freely (within a range of $\pm 5 \mathrm{~cm}$ ) along the vertical axis and in the horizontal plane (within $\pm 3 \mathrm{~cm})$.

\section{EXPERIMENTS}

The DJI F550 hexacopter was chosen for the final setup, which offered a good compromise in terms of maximum payload and limited downwash effect. In fact, the adoption of this gripping solution with a larger drone (DJI S900) was abandoned because the excessive downwash produced by the rotors tended to push away the bricks below.

The magnets chosen for the final implementation of the plate were $5 \mathrm{~mm}$ with a declared attraction force of approximately $3.8 \mathrm{~kg}$ for each single magnet, as shown in Table 1 . The nominal value of the force of attraction is confirmed if the object is made of iron. For steel or other alloys, this value can be reduced by more than $30 \%$. Often, this given value decreases due to the coating or surface irregularities of the magnets. Another factor is the thickness of the metal surface to be lifted, which must not be too thin, or the force of attraction cannot be fully exploited. However, in this study, the presence of the ULTRAT air gap between the magnet and the ferrous surface to be lifted led to a considerable deterioration in the attraction force, which decreased very rapidly with increasing distance. Ten magnets of the above model were inserted into the plate, as shown in Figure 2 , with alternating orientation in the magnetic field. Conducting the tensile test with the aid of a digital dynamometer, it was necessary to apply a force of $4.9 \mathrm{~kg}$ to detach the plate from the thick ferrous surface $(0.5 \mathrm{~cm})$. Given the above considerations and the degradation in the attraction obtained for each individual magnet, this force was still well within the acceptable limits. The use of passive magnets ensured a strong grip without any detachments during the transport phases despite the thin separation layer between the ferromagnetic surface and the magnets.

The final configuration was tested with copies of bricks, as per the challenge rules, with different weights of up to $2.0 \mathrm{~kg}$ and with thinner ferromagnetic surfaces $(0.1-0.2 \mathrm{~cm})$ on top compared with those used for the preliminary tensile test. During these tests, it was possible to lift the different types of bricks, but the orange bricks, shown in Figure 5, could not be lifted because the ferromagnetic surface was not thick enough to ensure a firm grip. However, since these bricks were $1.80-\mathrm{m}$ long, it might not be possible to lift them with just one drone, and therefore it was decided not to address this issue.

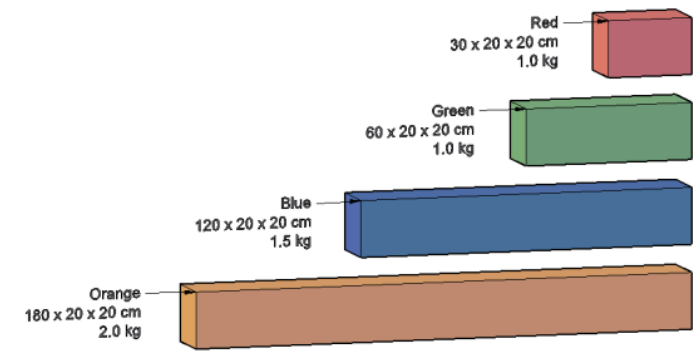

Figure 5. Types of bricks according to the challenge rules. 


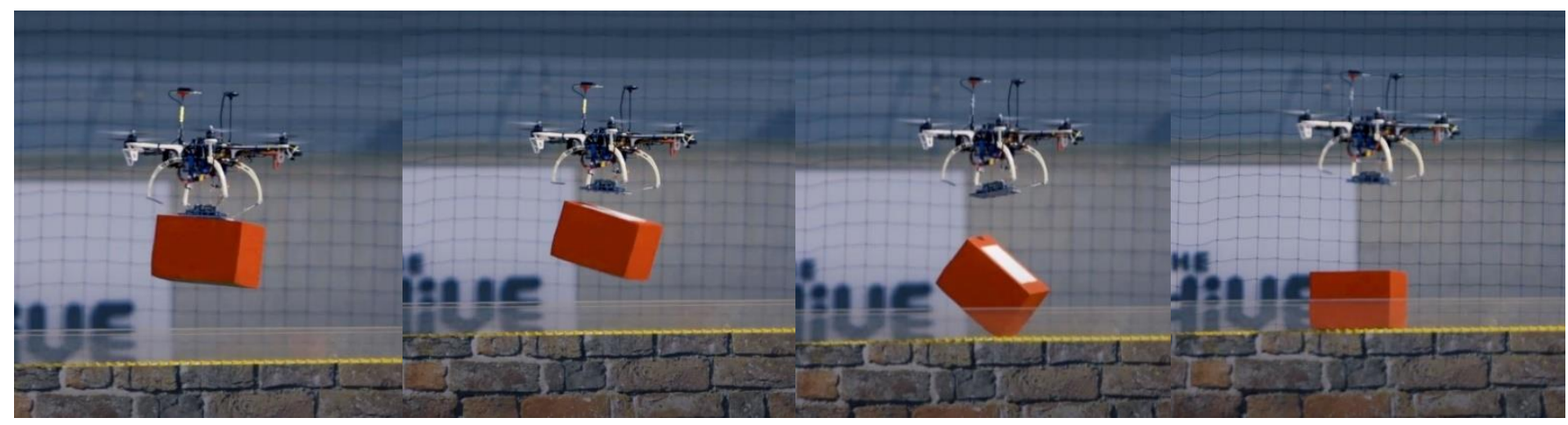

Figure 6 . The release phase, in which it is possible to see how the plate, thanks to the proposed solution, returns to its original position immediately after release without affecting the posture of the drone.

The release occurs by activating the servomotor, which produces an increase in the air gap (Figure 3) between the magnets and the metal surface of the gripped object. The detachment occurs naturally due to the contribution of gravity, so a small servo can detach objects in the order of $1-2 \mathrm{~kg}$. This means that as the weight of the object decreases, it is necessary to increase the air gap produced by the movement of the levers. A short release sequence is shown in Figure 6.

This magnetic plate is presented in [6], and in the current version it has been improved with the integration of a sheet of magnetic shield material. This is an alloy with good magnetic permeability that allows the shielding of magnetic fields. This solution allows the upper part of the plate to be shielded, thus avoiding any interference with the autopilot and other system components.

The proposed system, as shown in Figure 7, allows excellent pick-and-place missions to be conducted. Furthermore, the decision not to use electromagnets avoids the intermittent generation of magnetic fields, which could negatively influence the behaviour of the autopilot and reduce flight times as a result of the power consumption during the gripping phase.

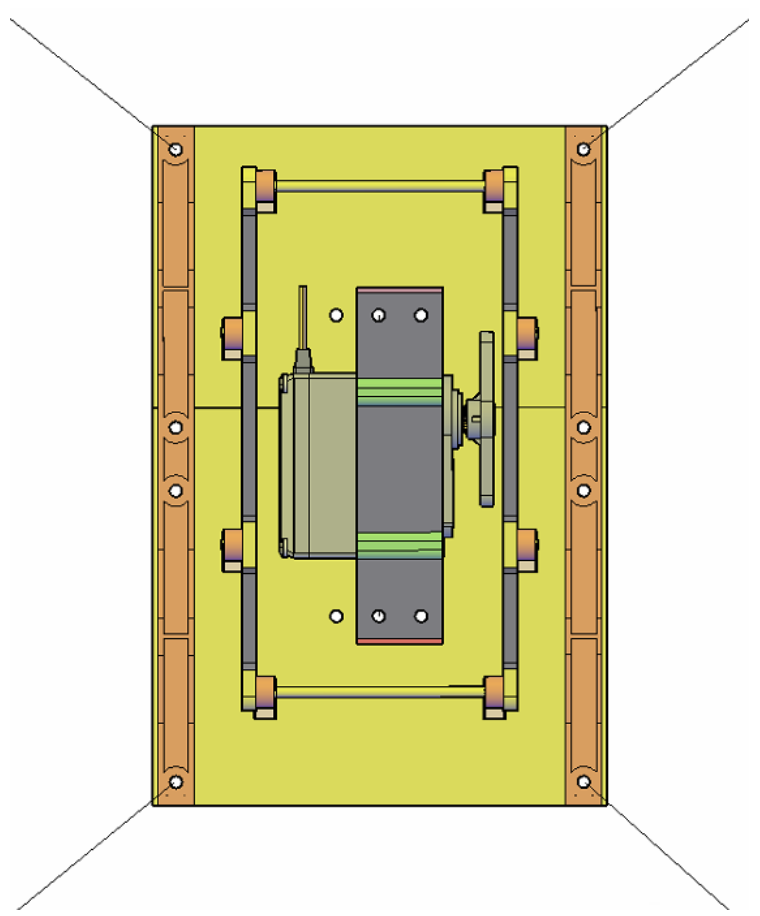

Figure 7. Final assembly with the damping system and cable tensioner.
The final assembly with the damping system with cables and springs guarantees a firm grip between the brick and the magnetic plate, dampening the vibrations caused by transportation. The tension springs chosen for this project increase the extension length by $5 \mathrm{~cm}$; as soon as the plate is within a range of $5 \mathrm{~cm}$, it is attracted to the ferrous surface and stretches, leading to automatic coupling. When the springs are fully extended, the drone is able to lift the brick gradually. Moreover, our damping system provides a compensation to payload vertical accelerations and decelerations respectively during take-off and landing.

The tests performed on the gripper during the challenge showed that it was able to grab, transport and place a large number of bricks during the time allowed for the trials.

\section{CONCLUSIONS}

In this article, a drone equipped with a pick-and-place system for objects with ferrous surfaces has been presented, and the different approaches used to carry out the delivery service using drones have been evaluated. Based on this analysis, it was decided to proceed using the technique of passive permanent magnets in order to eliminate the power consumption during the transport phase. This technique has been combined with a custom design in order to obtain a flat profile and to guarantee the lightness of the prototype as a result of using $3 \mathrm{D}$ printing. From the literature, it appears that this model represents the most compact passive magnetic gripping system developed.

In the future, a lighter and flexible version of the prototype, capable of lifting objects with limited curved faces, will be developed. Moreover, as the current vision system for brick detection is placed under an arm, the field of view is partially hidden by the plate. Therefore, as a future development, the camera will be integrated into the plate in order to improve visualisation and ensure alignment up to a few centimetres from the object to be grasped. In addition, distance sensors will be installed to constantly monitor the distance during the gripping phase.

Based on the positive results from MBZIRC and the latest improvements, this drone can be employed in the field in case of emergency to transport goods in dangerous or remote areas.

\section{ACKNOWLEDGEMENT}

This research was partially funded by the 'Safe and Smart Farming with Artificial Intelligence and Robotics - programma ricerca di ateneo UNICT 2020 -- 22 linea 2' project. 


\section{REFERENCES}

[1] D. Longo, G. Muscato, Adhesion techniques for climbing robots: state of the art and experimental considerations, Advances in Mobile Robotics, Proc. of the Eleventh International Conference on Climbing and Walking Robots and the Support Technologies for Mobile Machines (CLAWAR), Coimbra, Portugal, 8 - 10 September 2008, pp. 6-28. DOI: 10.1142/9789812835772 0003

[2] A. Rodríguez Castaño, F. Real, P. Ramón Soria, J. Capitán Fernández, V. Vega, B. C. Arrue Ullés, A. Ollero Baturone, Alrobotics team: a cooperative multi-unmanned aerial vehicle approach for the Mohamed Bin Zayed International Robotic Challenge, Journal of Field Robotics 36 (2019) pp. 104-124. DOI: $10.1002 /$ rob. 21810

[3] A. Gawel, M. Kamel, T. Novkovic, J. Widauer, D. Schindler, B. P. Von Altishofen, R. Siegwart, J. Nieto, Aerial picking and delivery of magnetic objects with mavs, Proc. of the 2017 IEEE International Conference on Robotics and Automation (ICRA),
Singapore, 29 May-3 June 2017, pp. 5746-5752.

DOI: $10.1109 /$ ICRA.2017.7989675

[4] K. Tai, A. R. El-Sayed, M. Shahriari, M. Biglarbegian, S. Mahmud, State of the art robotic grippers and applications, Robotics 5 (2016). 20 pp.

DOI: $10.3390 /$ robotics 5020011

[5] C. G. Hooi, F. D. Lagor, D. A. Paley, Height estimation and control of rotorcraft in ground effect using spatially distributed pressure sensing, Journal of the American Helicopter Society 61 (2016) pp. 1-14.

DOI: $10.4050 / \mathrm{JAHS} .61 .042004$

[6] G. Sutera, D. C. Guastella, G. Muscato, A novel design of a lightweight magnetic plate for a delivery drone, Proc. of the 23rd International Symposium on Measurement and Control in Robotics (ISMCR), Budapest, Hungary, 15-17 October 2020, pp. 1-4.

DOI: $10.1109 /$ ISMCR 51255.2020.9263730 